ARTÍ́CULO

\title{
Efecto de la Fertilización Nitrogenada y Fosforada en plantas de Raulí sobre su Respuesta Fisiológica, Crecimiento, Producción de Brotes y Enraizamiento de Estacas.
}

\author{
Hernández, José *1, Quiroz, Iván 1, Pincheira, Matías ² y Gacitúa, Sandra ${ }^{1}$ \\ ${ }^{1}$ Instituto Forestal Chile. jhernandez@infor.cl \\ ${ }^{2}$ CMPC- Desarrollo Silvícola \\ *Autor de correspondencia:
}

\begin{abstract}
RESUMEN
En Chile, existen extensas superficies de monocultivo establecidas con las especies de Pinus sp y Eucalyptus sp, por ello surge la necesidad de procurar la diversificación de las especies. Raulí (Nothofagus alpina (Poepp et Endl) Oerst $\sim N$. nervosa (Phil.) Dim. et Mil) es una especie con potencialidad comercial, cuyo rápido crecimiento, calidad y uso de la madera hacen que se le considere como una alternativa económica para el país. En este contexto, el objetivo del estudio fue determinar el efecto de la fertilización con diferentes niveles de nitrógeno y fósforo en la respuesta morfofisiológica presentada por raulí creciendo en vivero. Se estudió el crecimiento de las plantas sometidos a distintos niveles de nitrógeno $(0,100,300$ y $500 \mathrm{mg} / \mathrm{L})$ en combinación factorial con fósforo $(0,50,150$ y $250 \mathrm{mg} / \mathrm{L})$. Se realizaron mediciones de diámetro de cuello $( \pm 0,1 \mathrm{~mm})$, altura $( \pm 0,1 \mathrm{~cm})$, área foliar $\left( \pm 0,1 \mathrm{~cm}^{2}\right)$, biomasa aérea $( \pm 0,1 \mathrm{~g})$, conductividad eléctrica del medio de crecimiento $(\mu \mathrm{S} / \mathrm{m})$ y fotosíntesis neta $\left(\mu \mathrm{mol} \mathrm{CO}_{2} / \mathrm{m}^{2} \mathrm{~s}\right)$. El aumento en la concentración de nitrógeno a $500 \mathrm{mg} / \mathrm{L}$ incorporada al medio de crecimiento provocó un aumento en la conductividad eléctrica hasta los $1.994 \pm 186 \mu \mathrm{S} / \mathrm{cm}$ causando toxicidad, no existiendo un efecto del fósforo sobre esta variable. A mayor concentración de nitrógeno y fósforo (sobre 100 y $150 \mathrm{mg} / \mathrm{L}$, respectivamente) disminuyó la fotosíntesis neta a $8 \mu \mathrm{mol} \mathrm{CO}_{2} / \mathrm{m}^{2} \mathrm{~s}$, lo que se tradujo en una disminución en las tasas de crecimiento en diámetro, altura, biomasa aérea y área foliar. El aumento en las concentraciones de nitrógeno en el medio de crecimiento provocó una disminución en el número total de brotes de primer orden producidas por los setos, variando desde 12 brotes en el tratamiento $0 \mathrm{mg} / \mathrm{L}$ de N / $150 \mathrm{mg} / \mathrm{L}$ de $P$ hasta 6 brotes en el tratamiento $500 \mathrm{mg} / \mathrm{L} \mathrm{de} \mathrm{N} / 0 \mathrm{mg} / \mathrm{L} \mathrm{de} P$, obteniendo un $75 \%$ de enraizamiento con el tratamiento $100 \mathrm{mg} / \mathrm{L}$ de $\mathrm{N} / 0$ y $50 \mathrm{mg} / \mathrm{L}$ de $P$.
\end{abstract}

Palabras clave: Fertilización, fotosíntesis, morfología, producción de brotes, enraizamiento.

\section{SUMMARY}

In Chile, there are extensive areas of monoculture established with the species of Pinus $s p$ and Eucalyptus $s p$, for this reason the need arises to ensure the diversification of the species. Raulí (Nothofagus alpina (Poepp et Endl) Oerst $\sim N$. nervosa (Phil.) Dim. et Mil) is a species with commercial potential for rapid growth, quality and use of wood, being considered as an economic alternative for the country. In this context, the aim of the study was to determine the effect of different levels of fertilization with nitrogen and phosphorus response by raulí morphophysiological growing in nursery. The growth of plants under different levels of nitrogen $(0,100,300$ and $500 \mathrm{mg} / \mathrm{L})$ in factorial combination with phosphorus $(0,50$, 150 and $250 \mathrm{mg} / \mathrm{L})$ was studied. Diameter measurements were made $( \pm 0.1 \mathrm{~mm})$, height $( \pm 0.1 \mathrm{~cm})$, leaf area $\left( \pm 0.1 \mathrm{~cm}^{2}\right)$, biomass $( \pm 0.1 \mathrm{~g})$, electrical conductivity of the growth medium $(\mu \mathrm{S} / \mathrm{m})$ and net photosynthesis $\left(\mu \mathrm{mol} \mathrm{CO}_{2} / \mathrm{m}^{2} \mathrm{~s}\right)$. The increase in the nitrogen concentration to $500 \mathrm{mg} / \mathrm{L}$ incorporated in the growth medium caused an increase in electrical conductivity up to $1.994 \pm 186 \mu \mathrm{S} / \mathrm{cm}$, causing toxicity, and there is no effect of phosphorus on this variable. A higher concentration of nitrogen and phosphorus (about 100 and $150 \mathrm{mg} / \mathrm{L}$, respectively) decreased net photosynthesis to $8 \mu \mathrm{mol} \mathrm{CO}_{2} / \mathrm{m}^{2} \mathrm{~s}$, which translates into a decrease in the rates of growth in diameter, height, biomass and area leaf. The increase in nitrogen concentrations in the growth medium caused a decrease in the total number of first-order shoots produced by the hedges, varying from 12 shoots in the treatment $0 \mathrm{mg} / \mathrm{L}$ of N/ $150 \mathrm{mg} / \mathrm{L}$ of $P$ up to 6 shoots in the treatment $500 \mathrm{mg} / \mathrm{L}$ of N/ $0 \mathrm{mg} / \mathrm{L}$ of $\mathrm{P}$, obtaining $75 \%$ rooting with the treatment $100 \mathrm{mg} / \mathrm{L}$ of $\mathrm{N} / 0$ and $50 \mathrm{mg} / \mathrm{L}$ of $\mathrm{P}$.

Key words: Fertilization, photosynthesis, morphology, shoots production, rooting. 


\section{INTRODUCCIÓN}

Las grandes extensiones de monocultivo en Chile sugieren la necesidad estratégica de propender a la diversificación de especies forestales (Lara et al., 2000). Tal situación, sumada a la creciente conciencia medioambiental y al interés por la madera nativa, constituye una alternativa para el establecimiento de plantaciones de especies nativas de alto valor ecológico y económico (Reyes et al., 2007).

Entre las especies nativas de importancia ecológica y económica se destaca el raulí (Nothofagus alpina (Poepp et Endl) Oerst N. nervosa (Phil.) Dim. et Mil), especie endémica de los bosques subantárticos de Chile y Argentina, que crece en las laderas de las montañas, a altitudes intermedias entre 400 y 1200 msnm (Donoso et al., 2006). En Chile, se distribuye por la Cordillera de los Andes desde el sur de la

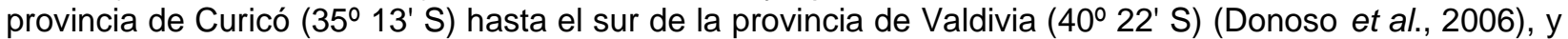
en la Cordillera de la Costa desde el norte de la Región del Maule (35둥' S) hasta la Región de los Lagos (411' S) (Sepúlveda y Stoll, 2003).

Raulí es considerada una de las especies con potencialidad de uso comercial, por su rápido crecimiento y calidad de la madera. En estudios de crecimiento realizados en plantaciones de 15 años provenientes de plantas colectadas de regeneración natural se ha observado incrementos diametrales anuales medios de $1 \mathrm{~cm}$, crecimientos periódicos de $1,5 \mathrm{~cm}$ y un crecimiento volumétrico medio de 12 a $17 \mathrm{~m}^{3} / \mathrm{ha} / \mathrm{año}$ (Donoso et al. 1993).

No obstante, los bajos estándares de calidad de planta (morfología y fisiología) de aquellos individuos recolectados directamente de la regeneración natural afectan directamente el potencial de crecimiento en terreno. En este sentido, Duryea \& Mcclain (1984) ya describen que el incremento en altura y diámetro de las plantas durante el primer año en terreno depende de su calidad y la interacción con las variables ambientales que definen las restricciones del sitio de establecimiento. De esta manera, prácticas como la fertilización en vivero cumplen un rol primordial para maximizar la calidad de planta y con ello mejorar su comportamiento en campo (Landis, 1985; Howell \& Harrington, 2004).

En este sentido, los programas regulares de fertilización con nitrógeno $(N)$ y fósforo $(P)$ son considerados esenciales para el mejoramiento de la calidad de plantas (Hernández y Rubilar, 2012). En la fisiología de una especie, la ausencia de nitrógeno y fósforo limita el crecimiento, restringe el desarrollo del área foliar y reduce la tasa fotosintética (Reich, 2009). Una baja disponibilidad de nitrógeno y fósforo provoca una restricción en la absorción de carbono (Marschner, 1995). El nitrógeno es un constituyente de proteínas, aminoácidos, ARN, ADN, y otras moléculas esenciales, además participa directamente en el proceso de la fotosíntesis, específicamente como constituyente de la ribulosa 1,5 bisfosfato carboxilasa oxygenasa (RuBP) (Nicodemus et al., 2008) y también favorece la regulación de la fosforilación (Flugge et al., 1980).

La eficiencia del uso del nitrógeno en la fotosíntesis se encuentra directamente correlacionada con la disponibilidad del fósforo en el medio, induciendo en conjunto el crecimiento de la planta. Una deficiencia de fósforo en los tejidos reduce la actividad tilacoidal estomática (Silvak \& Walker, 1986), modifica la fluorescencia clorofílica, la eficiencia de la luz (Conroy et al., 1986) y reduce la regeneración de la RuBP (Cambell \& Sage, 2006), lo que en suma se traduce en una reducción de la tasa fotosintética.

Estudios de fertilización en plántulas de Pinus elliotti muestran un incremento en el crecimiento como respuesta al aumento de las concentraciones de nitrógeno en el medio de crecimiento (Comerford \& Fisher, 1984). Imo \& Timmer (1992) determinaron que la fertilización nitrogenada incrementó el crecimiento de plantas de Prosopis chilensis entre 1047-1294\%. Resultados similares fueron obtenidos por Salifu \& Timmer (2003), quienes en Picea mariana lograron un incremento en crecimiento en altura entre $257-361 \%$. El fósforo es otro importante elemento en las plantas recién establecidas, tal así que aproximadamente el $75 \%$ del fósforo necesario para el desarrollo de nuevos tejidos se obtiene a través de la translocación de este nutriente desde las hojas producidas durante la fase en vivero (Van Den Driessche, 1991).

La fertilización afecta directamente el nivel nutricional de las plantas, y está altamente relacionado con el aumento de los niveles de carbohidratos, la producción de brotes y enraizamiento adventicio. Según Moe \& Andersen (1988), los esquemas de nutrición en los setos se orientan a la rápida respuesta de las estacas en formación y producción de raíces, modificando la cantidad relativa de nitrógeno y carbohidratos, para inducir el enraizamiento adventicio. Estos conceptos se basan en que una alta relación carbohidratos/nitrógeno estimula el enraizamiento de las estacas de tomate (Lycopersicon 
esculentum). Así mismo, la concentración de carbohidratos totales influenciada por la fertilización, afecta al enraizamiento mediante la entrega de energía de reserva y carbono que estimula el enraizamiento y posterior crecimiento.

En el caso de raulí, no existen antecedentes publicados del efecto de la fertilización nitrogenada y fosforada en los parámetros morfológicos de la planta, su respuesta en la fotosíntesis neta, producción de brotes y la respuesta en enraizamiento de sus estacas.

Consecuentemente, el objetivo de esta investigación fue determinar el efecto de la fertilización con diferentes niveles de nitrógeno y fósforo en la respuesta morfológica, fisiológica, producción de brotes y enraizamiento presentada en plantas de raulí durante su viverización. Lo anterior, basado en la hipótesis que el aumento lineal en las concentraciones de nitrógeno y fósforo en el medio de crecimiento favorece la fotosíntesis neta, traduciéndose en un mayor crecimiento en altura y diámetro y favoreciendo la producción de brotes y enraizamiento de las estacas.

\section{MATERIAL Y MÉTODO}

\section{Antecedentes Generales}

El estudio fue realizado en el vivero experimental del Centro Tecnológico de la Planta forestal, Instituto

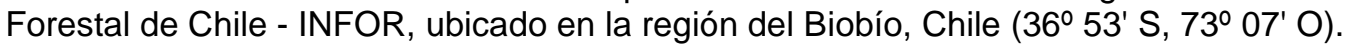

Se utilizó semillas con capacidad germinativa del $73 \%$, recolectadas del sector Vegas Blancas comuna de Angol (37ㄴ 49' S, 72 $53^{\prime}$ O). Se realizó un tratamiento pre-germinativo de las semillas consistente en su inmersión durante 24 horas en ácido giberélico, a concentración de 200 mg/L.

La siembra fue realizada a una temperatura ambiente de $25 \pm 1,0 \stackrel{\circ}{ } \mathrm{C}$, en almacigueras de poliestireno expandido de 84 cavidades, con un volumen por cavidad de $130 \mathrm{~cm}^{3}$.

Se utilizó sustrato de corteza compostada de Pinus radiata D. Don., con una porosidad total de 49,3\%, porosidad de aireación de $25,2 \%$ y porosidad de retención de $24,1 \%$; con un $39,5 \%$ de carbono orgánico y $0,6 \%$ de nitrógeno total; $766,2 \mathrm{mg} / \mathrm{Kg}$ nitrógeno amoniacal, y 7,0 mg/Kg nitrógeno nítrico. Trascurridos 14 días, las plántulas presentaban una altura de $1,0 \pm 0,5 \mathrm{~cm}$, momento que fueron trasplantadas a macetas de $1.000 \mathrm{~cm}^{3}$.

\section{Tratamientos de Fertilización}

Se consideró un diseño de bloques completos al azar con tres repeticiones, bajo un esquema factorial de cuatro concentraciones de nitrógeno $(0,100,300$ y $500 \mathrm{mg} / \mathrm{L})$ por cuatro concentraciones de fósforo $(0$, $50,150,250 \mathrm{mg} / \mathrm{L})$.

La unidad experimental estuvo compuesta por dos macetas, en cada una se distribuyó 6 plántulas en forma sistemática. La preparación de las soluciones nutritivas se realizó según la metodología propuesta por Landis (1985) (Figura 1).

Los tratamientos de fertilización se aplicaron semanalmente mediante ferti-riego, considerando primero la adición por separado de $500 \mathrm{ml}$ de solución de nitrato de calcio $\mathrm{Ca}\left(\mathrm{NO}_{3}\right)_{2}$ y $500 \mathrm{ml}$ de solución con los restantes elementos, a fin de evitar la precipitación del calcio con los sulfatos y fosfatos utilizados.

El aporte de elementos nutritivos del agua de riego se obtuvo mediante un análisis químico, el que determinó un aporte de calcio $(13,96 \mathrm{mg} / \mathrm{L})$, magnesio $(6,01 \mathrm{mg} / \mathrm{L})$, potasio $(4,26 \mathrm{mg} / \mathrm{L})$, nitrógeno amoniacal $(0,04 \mathrm{mg} / \mathrm{L})$ y fósforo $(0,04 \mathrm{mg} / \mathrm{L})$. Estos valores fueron incorporados al balance de las soluciones nutritivas elaboradas y aplicadas.

Adicionalmente, se verificó el pH del agua de riego $(7,26)$, siendo necesario su modificación de acuerdo al valor objetivo cifrado de 6,5 especificado en Landis et al. (1985). 


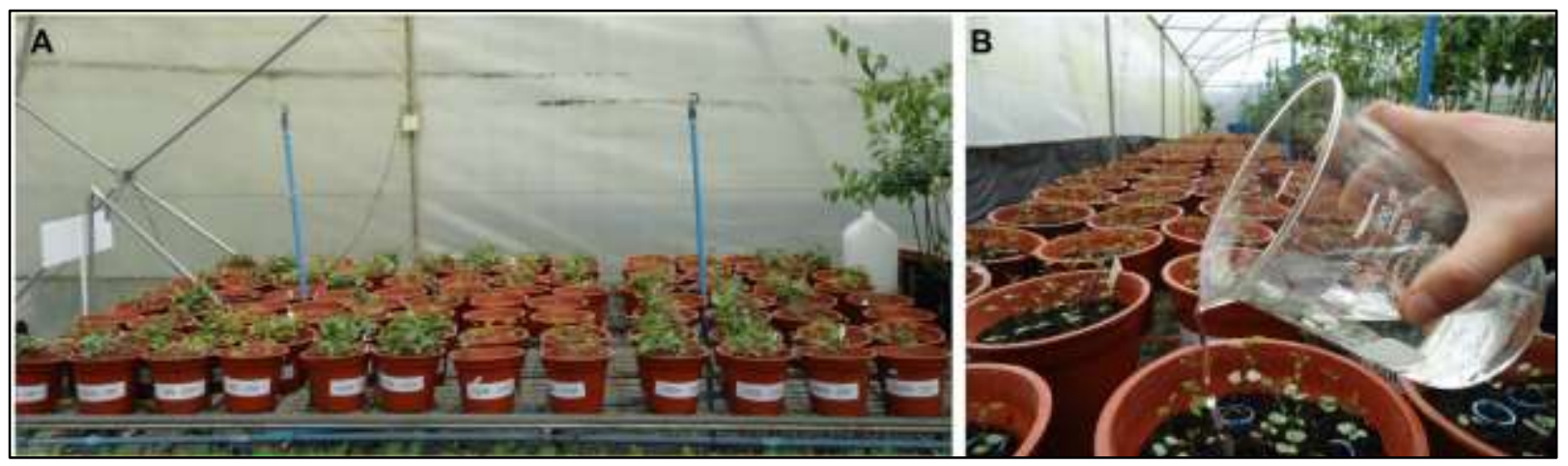

Figura 1. Distribución del ensayo (A) y fertilización de los tratamientos (B).

La frecuencia de riego fue establecida en función del porcentaje de humedad del sustrato. Se consideró una pérdida del $20 \%$ de la capacidad de campo, monitoreada diariamente a través de un sensor WET HH2 Read (Delta-T Devices Ltd). Para ello las macetas fueron regadas de forma manual a saturación (adicionando aproximadamente $1.000 \mathrm{ml}$ de agua).

Culminada la fase de viverización se registró, mediante un sensor WET HH2 Read (Delta-T Devices Ltd), la conductividad eléctrica del medio de crecimiento en cada uno de los tratamientos de nutrición establecidos.

\section{Parámetros Morfológicos de la Planta}

Finalizada la fase de viverización se seleccionó aleatoriamente tres plantas de cada unidad muestral. Cada planta seleccionada fue evaluada en diámetro a la altura del cuello $( \pm 0,1 \mathrm{~mm})$, altura $( \pm 0,1 \mathrm{~cm})$ y área foliar $\left(\mathrm{cm}^{2}\right)$. Los componentes de biomasa fueron separados y secados a $105 \pm 0,5 \stackrel{\circ}{\circ}$ hasta peso constante, usando una estufa de aíre forzado.

\section{Intercambio gaseoso.}

Desde cada una de las unidades muestrales se seleccionó al azar tres plantas del tratamiento control (0 $\mathrm{mg} / \mathrm{L}$ de $\mathrm{N}$ y $0 \mathrm{mg} / \mathrm{L}$ de $\mathrm{P}$ ), a ellas se les seleccionó una hoja del tercio superior para determinar la fotosíntesis neta, desde los 0 hasta los $2.000 \mu \mathrm{mol} \mathrm{CO} / \mathrm{m}^{2} \mathrm{~s}$ con un intervalo creciente de $100 \mu \mathrm{mol}$ $\mathrm{CO}_{2} / \mathrm{m}^{2} \mathrm{~s}$, mediante un equipo portátil de intercambio de gases (Walz Model 3040-L).

Con los valores de fotosíntesis obtenidos se ajustó una curva de respuesta de fotosíntesis a la luz, considerando el modelo de regresión no lineal de Michaelis-Menten de dos parámetros, el cual describe una forma hiperbólica rectangular (ec 1) (Coombs et al., 1985). El ajuste se efectuó mediante regresión no lineal utilizando el software InfoStat versión $2011 p$.

$$
A=\frac{(a * P A R)}{(b+P A R)}
$$

Donde,

A: Tasa fotosintética a las distintas intensidades de radiación $\left(\mu m o / \mathrm{CO}_{2} / \mathrm{m}^{2} \mathrm{~s}\right)$.

PAR: Intensidad de radiación $\left(\mu \mathrm{mol} \mathrm{m}^{-2} \mathrm{~s}^{-1}\right)$.

a: Parámetro indicador del valor de asimilación máxima (Amax).

b: Parámetro indicador del valor PAR en el que se alcanza $1 / 2$ Amax.

Adicionalmente, se seleccionó tres plantas por tratamiento y por unidad muestral ( 3 plantas $\times 3$ unidades muestrales $\times 16$ tratamientos), sobre las cuales se determinó fotosíntesis neta a 500 y $2000 \mu \mathrm{mol}$ $\mathrm{CO}_{2} / \mathrm{m}^{2} \mathrm{~s}$, valores definidos sobre los resultados obtenidos de la curva de luz de referencia (Figura 2). 


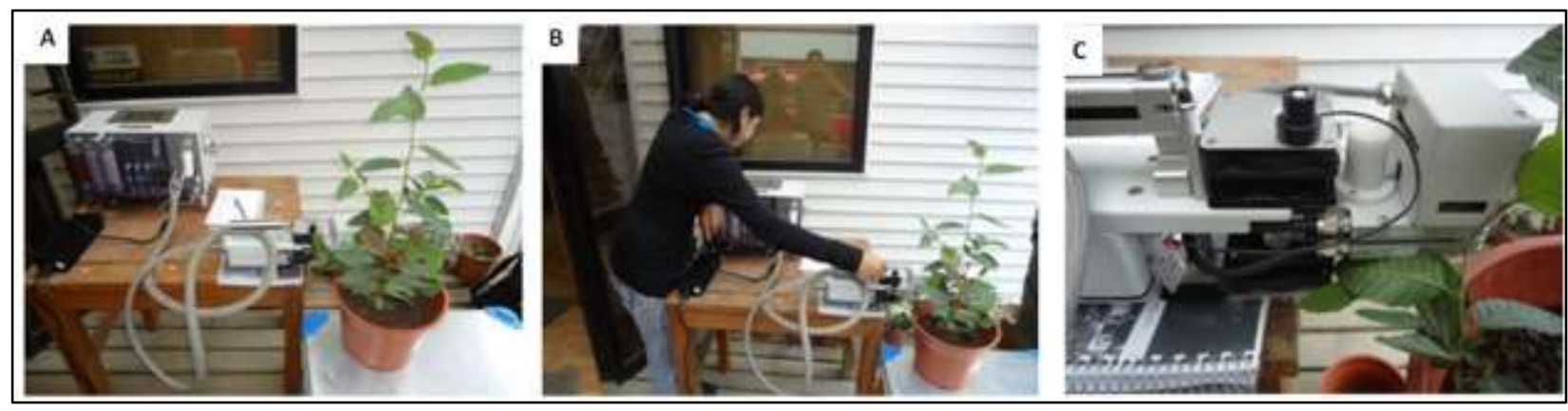

Figura 2. Medición de curva de capacidad fotosintética: Instalación del equipo (A), registro (B) y medición de los parámetros (C).

\section{Recolección y Establecimiento de Estacas en el Medio de Crecimiento}

Al final del periodo de crecimiento se colectó material desde plantas de cada uno de los tratamientos de fertilización aplicado. Se seleccionó al azar estacas semileñosas de la sección superior de las plantas, con una longitud de $7,0 \pm 1,0 \mathrm{~cm}$, diámetro de 3,0 $\pm 0,5 \mathrm{~mm}$, con presencia de al menos 2 yemas visibles y dejando como máximo un par de hojas en el tercio superior de la estaca. El corte basal se realizó en forma oblicua y fueron almacenadas en un recipiente con agua para evitar la desecación de las estacas durante su manipulación (Figura 3).

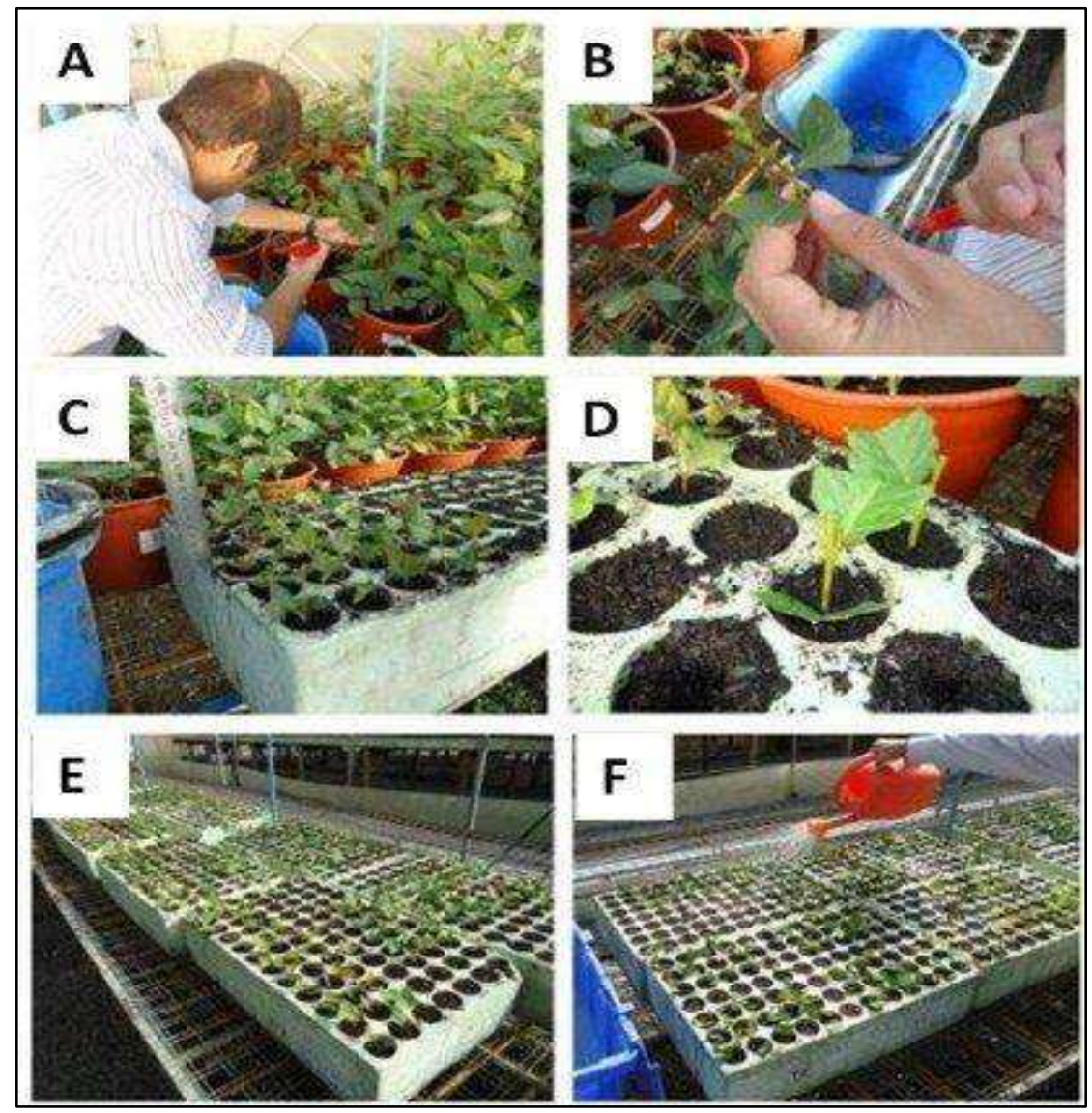

Figura 3. Recolección de estacas (A), preparación de la estaca (B), establecimiento de la estaca en el medio de crecimiento $(C D)$, estacas distribuidas aleatoriamente según su diseño $(E)$ y riego de las estacas $(F)$. 
El enraizamiento se efectuó en almacigueras de aislapol con cavidades de $16 \mathrm{~cm}$ de profundidad y volumen de $130 \mathrm{cc} \mathrm{cm}^{3}$. Se utilizó corteza compostada de pino como sustrato de enraizamiento y se usó riego tecnificado automático por aspersión mediante microjets. El programa de riego consideró la aplicación de tres riegos diarios de un minuto, manteniendo el sustrato a una humedad del $80 \%$.

\section{Análisis de Datos}

Culminado el período de crecimiento se evaluó, mediante análisis de varianza (ANDEVA), el efecto de la fertilización nitrogenada y fosforada en el crecimiento en altura, diámetro, biomasa de tallo, área foliar, biomasa de hoja, conductividad eléctrica, fotosíntesis neta, producción de brotes y enraizamiento. La homogeneidad de varianza fue evaluada mediante la prueba de Levene ( $p$-value $<0,05)$. El supuesto de normalidad de los residuos fue evaluado mediante la prueba de Shapiro-Wilks ( $p$ value $<0,05$ ). La significancia de las diferencias entre tratamientos se determinó mediante la prueba de comparación múltiple de Tukey-Kramer, con un $95 \%$ de confianza.

\section{RESULTADOS}

El crecimiento en altura y diámetro promedio al final del período de evaluación, varió significativamente $(p$ value $\leq 0,05)$ desde $15,4 \pm 1,9 \mathrm{~cm}$ y $3,5 \pm 0,2 \mathrm{~mm}$ en el testigo con $0 \mathrm{mg} / \mathrm{L}$ de nitrógeno y $0 \mathrm{mg} / \mathrm{L}$ de fosforo hasta $45,1 \pm 5,1 \mathrm{~cm}$ y $5,3 \pm 0,3 \mathrm{~mm}$ al fertilizar con $500 \mathrm{mg} / \mathrm{L}$ de nitrógeno y $50 \mathrm{mg} / \mathrm{L}$ de fosforo, provocando un aumento del 192 y $51 \%$ respectivamente (Figura 4). Las concentraciones de fósforo afectaron significativamente ( $p$ value $\leq 0,05$ ) ambos atributos morfológicos, variando desde 12,4 $\pm 1,9 \mathrm{~cm}$ y $3,5 \pm 0,7 \mathrm{~mm}$ con $0 \mathrm{mg} / \mathrm{L}$ hasta $25,7 \pm 4,1 \mathrm{~cm}$ y $3,9 \pm 0,6 \mathrm{~mm}$ al fertilizar con $250 \mathrm{mg} / \mathrm{L}$ de fósforo, provocando un aumento de 11 y $161 \%$, respectivamente. No existió interacción entre los dos elementos tanto para en el crecimiento en diámetro ( $p$ value $=0,377)$, como en altura ( $p$ value $=0,467$ ).

Al final del estudio la conductividad eléctrica del medio de crecimiento se incrementó significativamente $(p$ value $\leq 0,05)$ a medida que aumentó la concentración de nitrógeno, desde $1098 \pm 286 \mu \mathrm{S} / \mathrm{cm}$ con $0 \mathrm{mg} / \mathrm{L}$ hasta $1994 \pm 186 \mu \mathrm{S} / \mathrm{cm}$ al fertilizar con $500 \mathrm{mg} / \mathrm{L}$ de nitrógeno, provocando un aumento de $81,6 \%$. No se observó efecto significativo del fósforo $(p$ value $=0,186)$, aunque sí se detectó una interacción significativa entre ambos elementos ( $p$ value $=0,003$ ) (Figura 5).

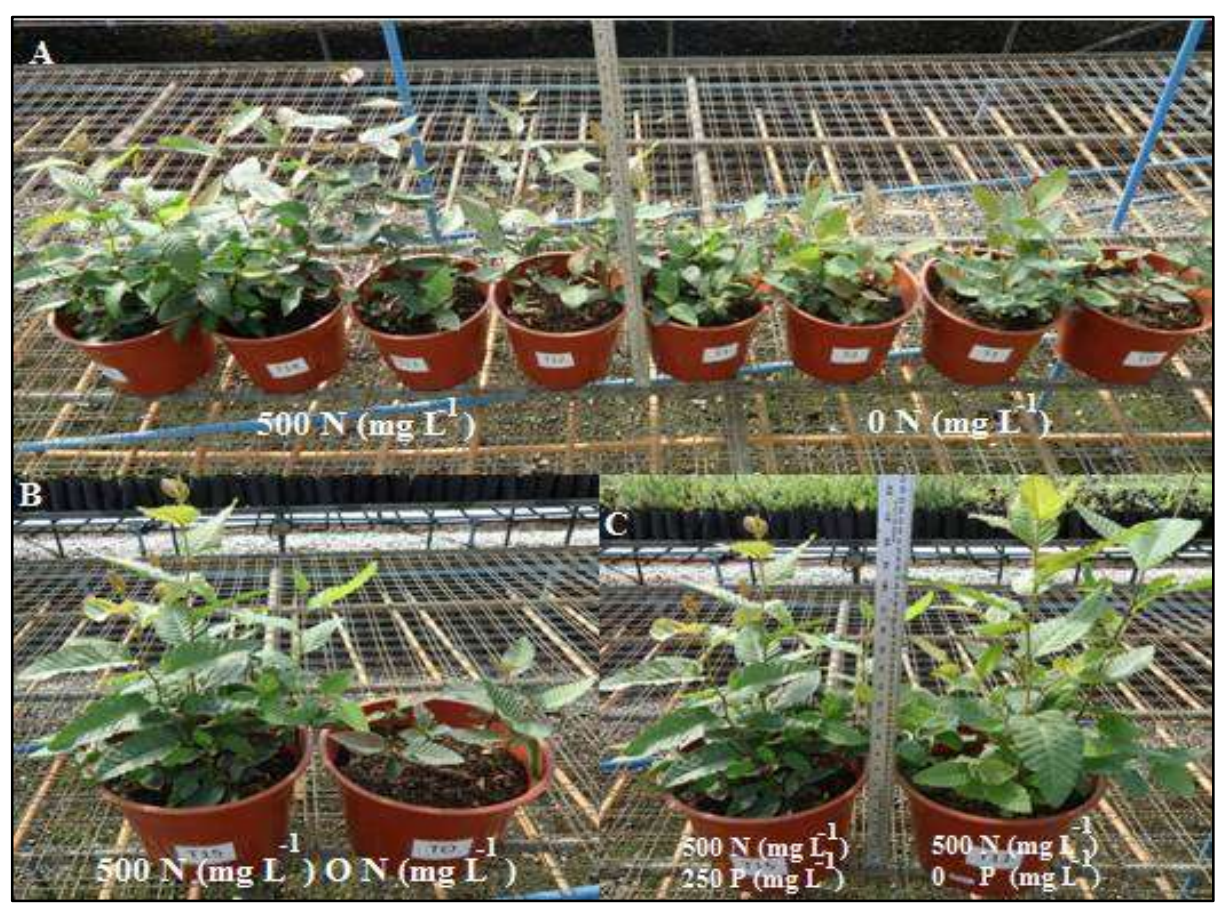

Figura 4. Efecto de la fertilización nitrogenada $(0,100,200$ y $400 \mathrm{mg} / \mathrm{L})$ y fosforada $(0,50,150$ y $250 \mathrm{mg} / \mathrm{L}$ ) en los parámetros morfológicos. 


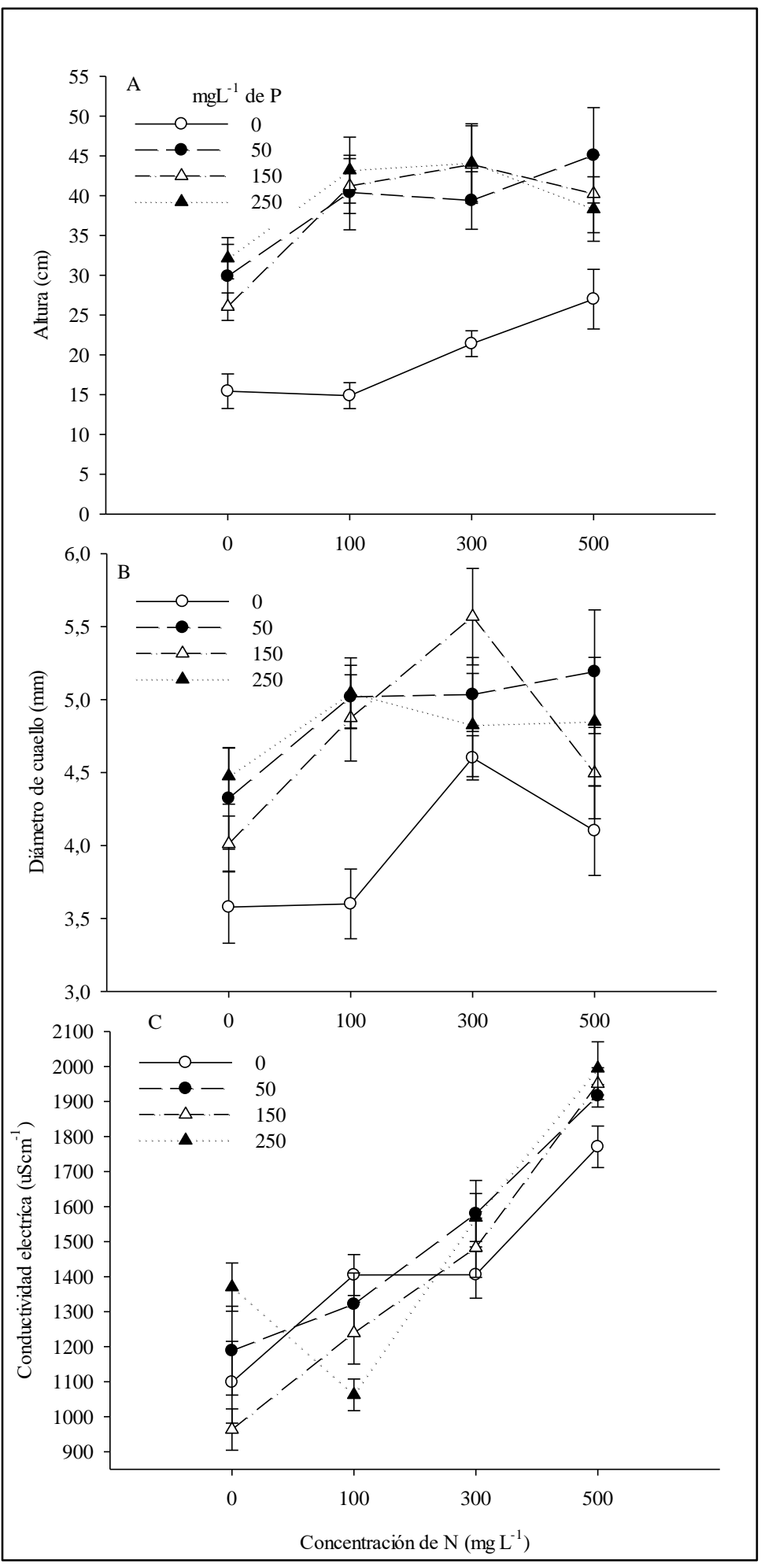

Figura 5. Efecto de la fertilización nitrogenada $(0,100,200$ y $400 \mathrm{mg} / \mathrm{L})$ y fosforada $(0,50,150$ y $250 \mathrm{mg} / \mathrm{L})$ al final del periodo de crecimiento. A) Altura. B) Diámetro de cuello. C) Conductividad electrica del medio de crecimiento. En plantas de raulí, valores corresponden a media \pm el error estándar $(n=9)$. 


\section{Biomasa Aérea y Área Foliar}

La biomasa aérea y área foliar obtenida al final del período de evaluación se incrementó significativamente $(p$ value $<0,001)$ con $100 \mathrm{mg} / \mathrm{L}$ y $300 \mathrm{mg} / \mathrm{L}$ de nitrógeno incorporados en el medio de crecimiento, variando desde $1,2 \pm 0,28 \mathrm{~g}$ y $165 \pm 51 \mathrm{~cm}^{2}$ con $0 \mathrm{mg} / \mathrm{L}$ hasta $4,0 \pm 1,4 \mathrm{~g}$ y $557,9 \pm 7,8 \mathrm{~cm}^{2}$ al fertilizar con 100 y $300 \mathrm{mg} / \mathrm{L}$ de nitrógeno, provocando un aumento de 233 y 238\%, respectivamente (Figura 6).
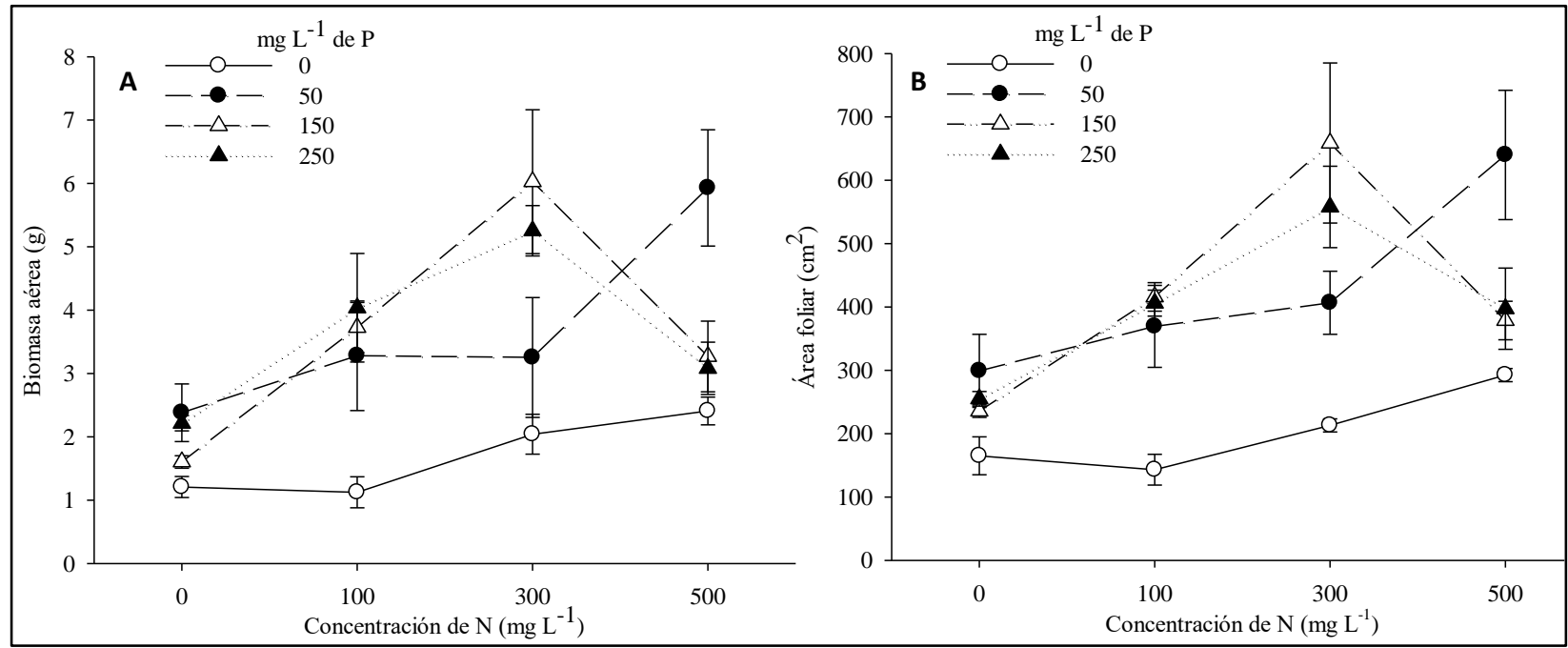

Figura 6. Efecto de la fertilización nitrogenada $(0,100,200$ y $400 \mathrm{mg} / \mathrm{L})$ y fosforada $(0,50,150$ y $250 \mathrm{mg} / \mathrm{L})$ al final del periodo de crecimiento. A) Biomasa aérea. B) Área foliar. En plantas de raulí, valores corresponden a media \pm el error estándar $(n=9)$.

\section{Intercambio Gaseoso}

Sobre la respuesta fotosintética a variaciones PAR se observó una asíntota superior de $13,8 \mu \mathrm{mo} / \mathrm{CO}_{2} /$ $\mathrm{m}^{2} \mathrm{~s}$ a los $2000 \mu \mathrm{mol} \mathrm{CO} / \mathrm{m}^{2} \mathrm{~s}$ (figura 7). La curva se caracterizó por una primera fase creciente y lineal que demuestra la eficiencia en la utilización de la luz por los cloroplastos, con una pendiente inicial de $0,05 \frac{\mathrm{CO}_{2} \mathrm{~m}^{2} \mathrm{~s}^{2}}{\mu \mathrm{mol} \mathrm{m} \mathrm{m}^{-2} \mathrm{~s}^{-1}}$ representando la fase donde la aceleración del proceso cambia de signo pasando de una velocidad creciente a una decreciente. Una segunda fase donde la pendiente tiende a un valor de 0 $\frac{\mathrm{CO}_{2} \mathrm{~m}^{2} \mathrm{~s}^{2}}{\mu \mathrm{mol} \mathrm{m} \mathrm{m}^{-2} \mathrm{~s}^{-1}}$ donde el rendimiento de flujo de fotones disminuye hasta un valor constante de fotosíntesis.

A los $9 \mu \mathrm{mol} \mathrm{CO} / \mathrm{m}^{2} \mathrm{~s}$ de densidad de flujo de fotones no existió una asimilación de fotosíntesis neta, igualándose las pérdidas de carbono por respiración y la fijación fotosintética. Al final del periodo de crecimiento, la fotosíntesis incrementó significativamente en función del aumento de la concentración de nitrógeno $(p$ value $\leq 0,05)$ y la concentración de fósforo $(p$ value $=0,001)$.

Se observó una alta interacción entre los elementos ( $p$ value $=0,006)$, hasta los $100 \mathrm{mg} / \mathrm{L}$ de nitrógeno y $50 \mathrm{mg} / \mathrm{L}$ de fósforo (figura 7). La fotosíntesis varió desde $3,6 \mu \mathrm{mo} / \mathrm{CO}_{2} / \mathrm{m}^{2} \mathrm{~s}$ con $50 \mathrm{mg} / \mathrm{L} \mathrm{de} \mathrm{P}$ y $0 \mathrm{mg} / \mathrm{L}$ de $\mathrm{N}$ hasta $12,5 \mu \mathrm{mol} \mathrm{CO}_{2} / \mathrm{m}^{2} \mathrm{~s}$ con $0 \mathrm{mg} / \mathrm{L}$ de $\mathrm{P}$ y $100 \mathrm{mg} / \mathrm{L}$ de $\mathrm{N}$, induciendo un aumento de $347 \%$ en la capacidad fotosintética en la especie.

Contrastantemente, el aumento en la concentración sobre los niveles de $100 \mathrm{mg} / \mathrm{L}$ de $\mathrm{N}$ y $50 \mathrm{mg} / \mathrm{L}$ de $P$ en la solución nutritiva no presentó efecto sobre en la capacidad fotosintética ( $p$ value $>0,05)$ (Figura 7 ). 


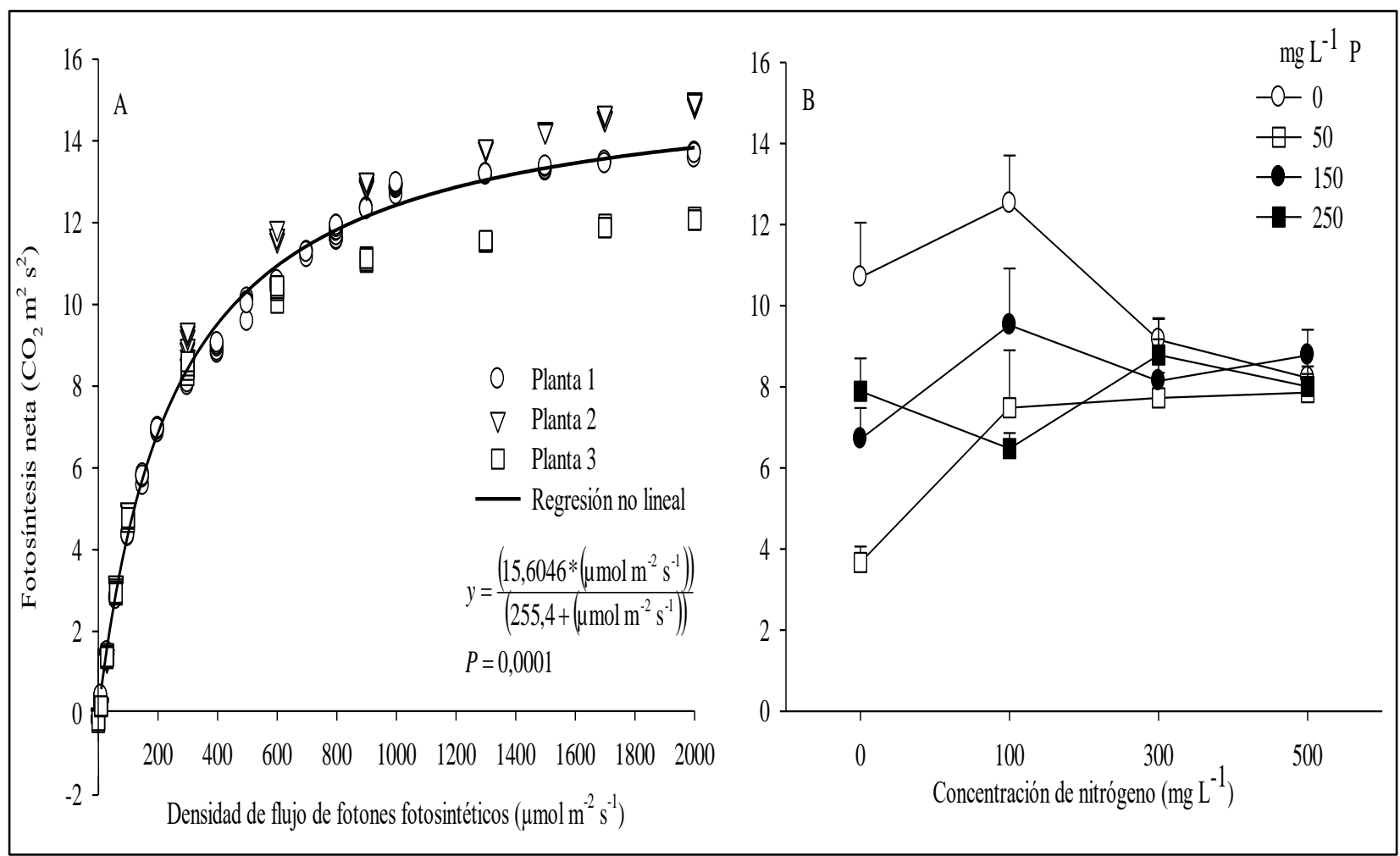

Figura 7. Tasas de intercambio gaseoso al finalizar el periodo de crecimiento, en plantas de raulí. A) Tasa fotosintética neta (Pn) a diferentes intensidades de luz (densidad de flujo fotónico), para el tratamiento control $(0 \mathrm{mg} / \mathrm{L} \mathrm{de} \mathrm{N}, 0 \mathrm{mg} / \mathrm{L}$ de $\mathrm{P})$. Datos observados $(\circ, \Delta, \square)$ y predicho (-). B) Tasa fotosintética neta (Pn) para el total de las concentraciones de $\mathrm{N}(0,100,300$ y $500 \mathrm{mg} / \mathrm{L})$ y $\mathrm{P}(0$, $50,150$ y $250 \mathrm{mg} / \mathrm{L})$. Valores corresponden a media \pm el error estándar $(n=9)$.

\section{Producción Total de Brotes y Enraizamiento de Estacas de Primer y Segundo Orden}

El aumento en las concentraciones de nitrógeno en el medio de crecimiento provocó una disminución en el número total de brotes de primer orden producidas por los setos, variando desde 12 brotes en el tratamiento $0 \mathrm{mg} / \mathrm{L}$ de $\mathrm{N} / 150 \mathrm{mg} / \mathrm{L}$ de $\mathrm{P}$ hasta 6 brotes en el tratamiento $500 \mathrm{mg} / \mathrm{L}$ de N $/ 0 \mathrm{mg} / \mathrm{L}$ de $P$, existiendo una disminución del $50 \%$ en el total de brotes producidos (Figura 8).

Al evaluar el efecto de la fertilización en el enraizamiento de las estacas estas variaron desde un $75 \%$ en el tratamiento $100 \mathrm{mg} / \mathrm{L}$ de N / 0 y $50 \mathrm{mg} / \mathrm{L}$ de $\mathrm{P}$ hasta un $38 \%$ en el tratamiento $500 \mathrm{mg} / \mathrm{L}$ de N / 250 $\mathrm{mg} / \mathrm{L}$ de $\mathrm{P}$, constatándose una disminución en el enraizamiento de un $37 \%$ a medida que aumentan las concentraciones de nitrógeno.

Al analizar el efecto de la fertilización nitrogenada y fosforada sobre el número total de estacas de segundo orden producidas, no se observa efecto de las concentraciones de nitrógeno ni de fósforo en esta variable ( $p$ value $>0,05)$.

Respecto al enraizamiento de estacas de primer y segundo orden, en ambos casos el mayor porcentaje de enraizamiento se obtuvo con la concentración de $100 \mathrm{mg} / \mathrm{L}$ de N / 50 mg/L de P (Figura 9). 


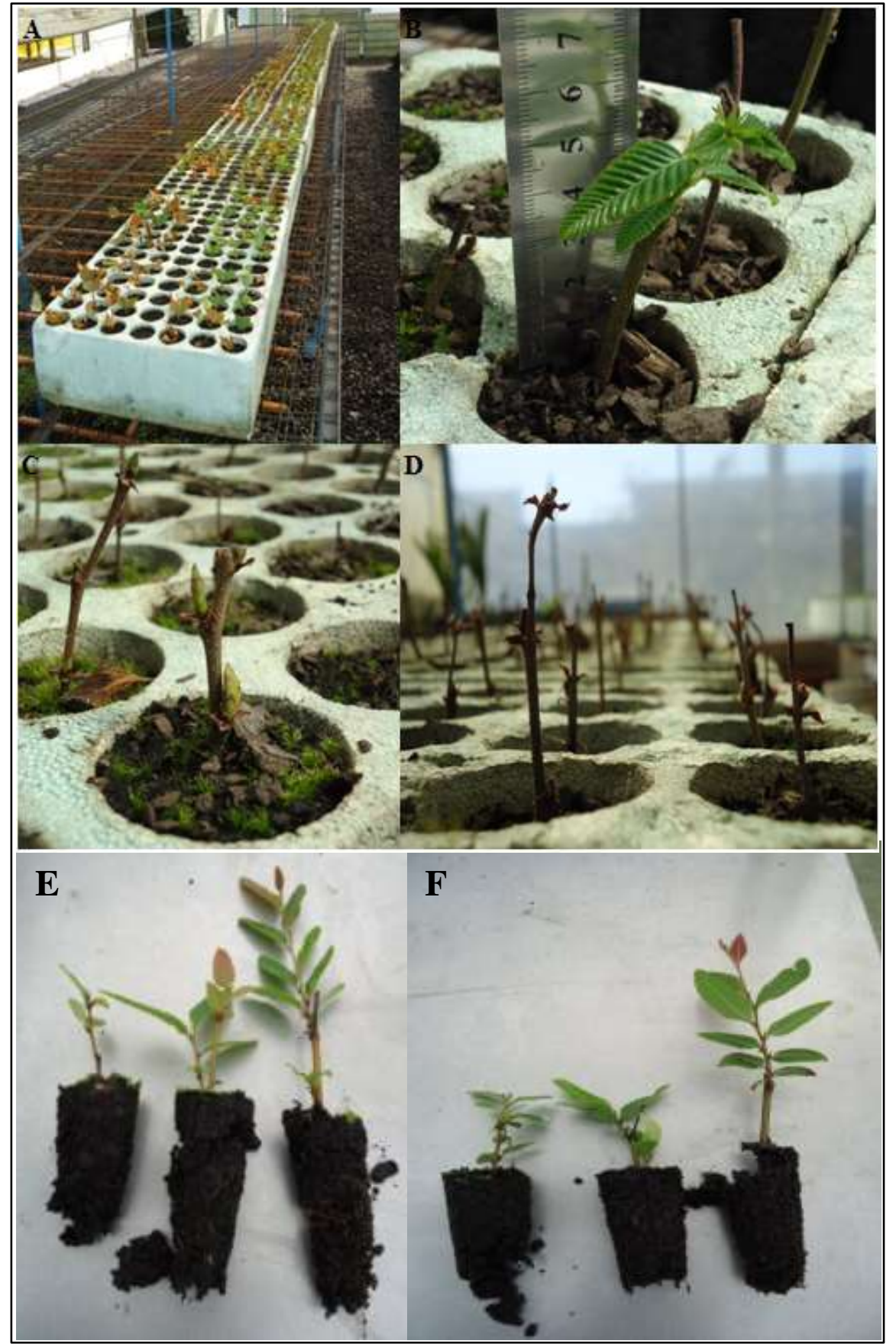

Figura 8. Efecto de la fertilización nitrogenada $(0,100,300$ y $500 \mathrm{mg} / \mathrm{L})$ y fosforada $(0,50,150$ y $250 \mathrm{mg} / \mathrm{L}$ ) en el enraizamiento de las estacas establecidas en el medio de crecimiento durante la estación de otoño: Establecimiento del ensayo de enraizamiento A); estacas enraizadas (B); brotación de las estacas (C); mortalidad de estacas (D); enraizamiento de estacas de primer orden (E) y enraizamiento de estacas de segundo orden $(F)$. 


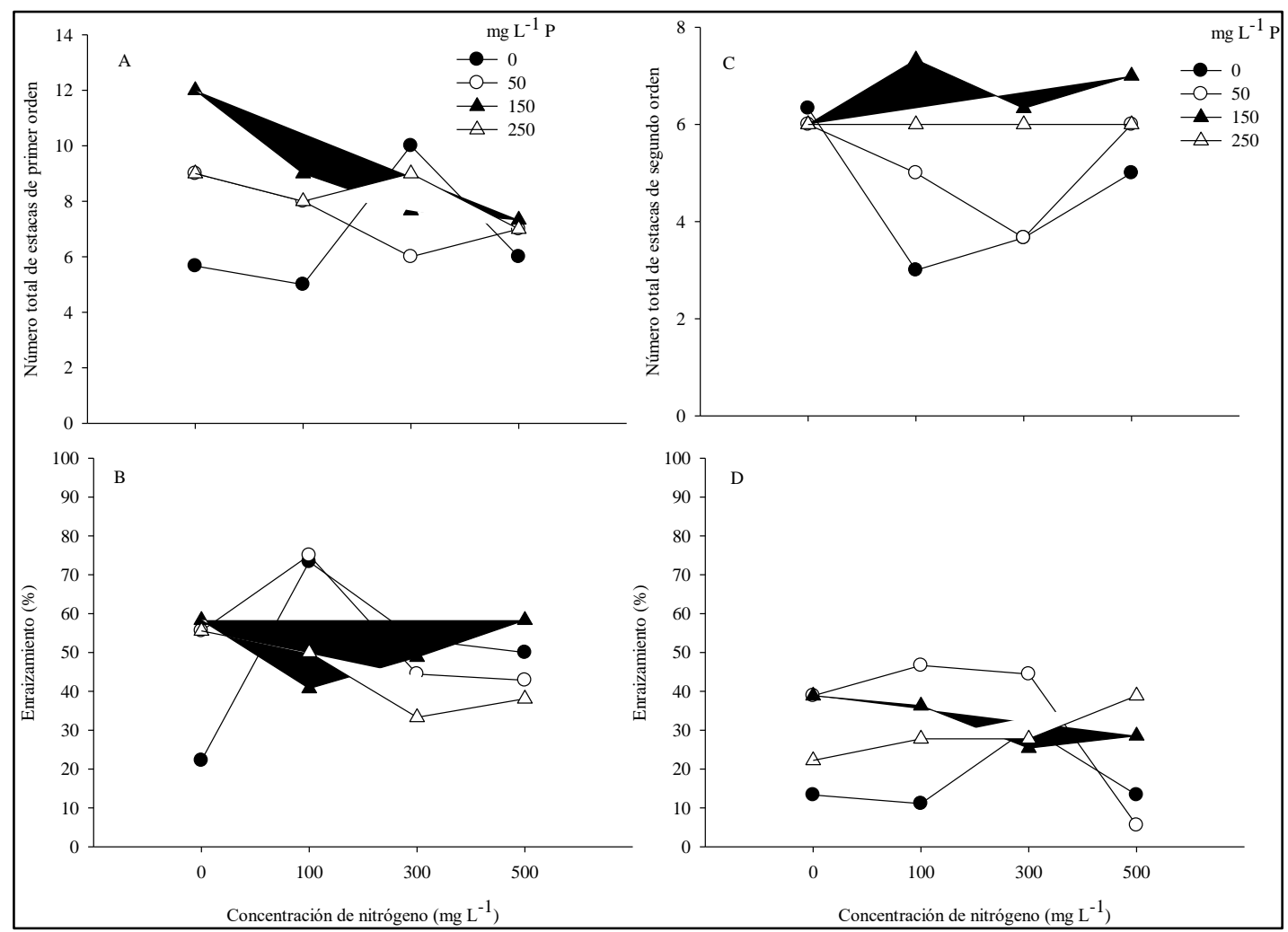

Figura 9. Efecto de la fertilización nitrogenada $(0,100,300$ y $500 \mathrm{mg} / \mathrm{L})$ y fosforada $(0,50,150$ y $250 \mathrm{mg} / \mathrm{L})$ en el número total de estacas de primer y segundo orden producidas, y el porcentaje de sobrevivencia asociado: Número total de estacas de primer orden producidas (A); porcentaje de enraizamiento en estacas de primer orden (B); número total de estacas de segundo orden (C) y porcentaje de enraizamiento en estacas de segundo orden (D).

\section{DISCUSIÓN}

Setos de raulí cultivados en macetas, usando corteza compostada de pino como sustrato, presentaron diversas respuestas a los tratamientos de fertilización, siendo explicadas satisfactoriamente por el modelo de crecimiento Michaelis-Menten de dos parámetros, que logra describir la relación entre la absorción de nutrientes, el crecimiento de las plantas y el esquema de fertilización, descrito previamente por Salifu \& Timmer (2003) para diferenciar la deficiencia de nutrientes, la suficiencia, el consumo de lujo y la toxicidad en plantas de Picea mariana.

El crecimiento en diámetro y altura de las plantas incrementaron significativamente a medida que aumentaron las concentraciones de nitrógeno y fósforo hasta los $100 \mathrm{mg} / \mathrm{L}$ de N / $50 \mathrm{mg} / \mathrm{L}$ de $\mathrm{P}$ (periodo de deficiencia), respuesta que se explica por la disponibilidad de nutrientes minerales (Landis, 2000). El crecimiento denotó estabilización sobre el tratamiento de $300 \mathrm{mg} / \mathrm{L}$ de $\mathrm{N} / 50 \mathrm{mg} / \mathrm{L}$ de $\mathrm{P}$ (periodo de consumo excesivo) producto del aumento en el contenido de nutrientes minerales presentes en el medio de crecimiento. Eventualmente, el crecimiento declinó debido a las altas tasas de nitrógeno y fósforo aplicadas sobre el tratamiento de $500 \mathrm{mg} / \mathrm{L}$ de N/150 mg/L de $\mathrm{P}$, observándose toxicidad en las plantas.

Los resultados obtenidos en este estudio ratifican lo planteado por Imo \& Timmer (1992), quienes al contrastar plantas de Prosopis chilensis no fertilizadas, con aquellas fertilizadas con $200 \mathrm{mg} / \mathrm{L}$ de nitrógeno, establecieron que la fertilización provocó un incremento en la altura de las plantas entre 1047 y $1294 \%$ ( $p$ value $<0,001$ ). Esto coincide también con lo observado por Hernández \& Rubilar (2012), quienes a una concentración de $400 \mathrm{mg} / \mathrm{L}$ de nitrógeno en el medio de crecimiento, determinaron un efecto positivo en la elongación de los brotes de $P$. radiata, correspondiente a un $93 \%$ de mayor crecimiento, respecto al tratamiento control (no fertilizado). Así mismo, investigaciones realizadas en Pinus halepensis determinaron que el crecimiento en altura de las plantas en vivero aumentó 
significativamente ( $p$ value $<0,001$ ) en un $310 \%$ y la supervivencia en un $37 \%$ en plantas que fueron fertilizadas, con respecto al tratamiento control (no fertilizado) (Oliet et al., 2009).

Del mismo modo, el incremento en crecimiento observado en respuesta a una alta disponibilidad de fósforo en el medio de crecimiento, concuerda con lo reportado para varias especies arbóreas establecidas en campo o en condiciones artificiales (Chang, 2003). Diversos autores sugirieren un efecto positivo del fósforo en el desarrollo de las raíces (Salisbury \& Ross, 2000). Plantas de Pseudotsuga menziesii fertilizadas con nitrógeno y fósforo presentaron un incremento significativo en la biomasa total y en el desarrollo de raíces en terreno (Bigg \& Schalau, 1990). Domínguez et al. (2000) indican que plantas de Pinus pinea en vivero responden a una mayor disponibilidad de fósforo presentando un mayor número de raíces, y posteriormente una alta capacidad de crecimiento de raíces al ser establecidas en terreno. Antecedentes establecidos bajo la premisa de que un mayor volumen radical de las plantas producidas en vivero favorecerá una mayor capacidad de absorción de agua y nutrientes, y por ende un mayor crecimiento inicial en terreno (Landis et al., 1985).

El aumento en la conductividad eléctrica por sobre $1.256 \pm 146 \mu \mathrm{s} / \mathrm{cm}$, como consecuencia de las altas concentraciones de nitrógeno incorporadas al medio de crecimiento, provocó una toxicidad que se manifestó en la disminución del crecimiento de las plantas; también demostró la alta sensibilidad de raulí a los cambios de concentraciones de nutrientes del medio de crecimiento. Estos mismos efectos han sido observados en otras especies (Hernández \& Rubilar, 2012).Los resultados obtenidos ratificaron lo planteado por Timmer \& Armstrong (1987), quienes establecieron que un aumento en la conductividad eléctrica producto de una saturación de fertilizantes en el medio de crecimiento afecta a la biomasa total de la planta, siendo posible la visualización de síntomas foliares, y la presencia de daños en el sistema radicular, asociados a cambios de la presión osmótica, producto de la toxicidad de cationes o aniones específicos (Thornton et al., 1988). En este sentido niveles óptimos de conductividad eléctrica asociados a la incorporación de nutrientes al medio de crecimiento, maximizarían la absorción y crecimiento de las plántulas; tal como se ha evidenciado en experimentos con plantas de Picea mariana y Picea glauca, las que presentaron su optimo crecimiento entre 1.200 y $2.500 \mu \mathrm{s} / \mathrm{cm}$ (Phillion \& Bunting, 1983). Similares resultados fueron obtenidos en Pinus resinosa obteniéndose su óptimo crecimiento entre 1.800 y 2.200 $\mu \mathrm{s} / \mathrm{cm}$ (Timmer \& Parton, 1984).

La fotosíntesis neta de las plantas se incrementó significativamente a medida que aumentaron las concentraciones de nitrógeno y fósforo, hasta alcanzar el intervalo de deficiencia de nutrientes $(100 \mathrm{mg} / \mathrm{L}$ de N / $50 \mathrm{mg} / \mathrm{L}$ de P) y coincidiendo con los resultados de crecimiento en altura y diámetro. Esto se debe a que a medida que aumentan las concentraciones de nitrógeno en la solución nutritiva, se incrementan las concentraciones de nitrógeno a nivel foliar (Hernández \& Rubilar, 2012), hasta el intervalo de periodo de consumo excesivo (Salifu \& Timmer, 2003). En este contexto, el incremento de la fotosíntesis producto de la fertilización provocó una mayor distribución de nitrógeno destinado a la producción de enzimas, encargadas en reaccionar con el carbono, aumentando las tasas de fijación del $\mathrm{CO}_{2}$ y traduciéndose en energía para la planta (Nicodemus et al., 2008). Así mismo, concentraciones adecuadas de fósforo incorporadas al medio de crecimiento provocaron un aumento en el crecimiento y fotosíntesis neta. Los resultados obtenidos ratifican lo planteado por Loustau et al. (1999), quienes determinaron que la nutrición fosforada afecta a la fotosíntesis principalmente a través de limitaciones químicas, tal como la disminución de la conductancia estomática, producto de la disminución del fósforo. Análogamente, tratamientos que sobrepasaron el intervalo de deficiencia, producto de una inadecuada concentración de fósforo, presentaron una disminución en la biomasa aérea de las plantas y en el área foliar. Esto es coincidente con lo planteado por Radin \& Eidenbock (1984), quienes demostraron que plantas de algodón disminuyeron el área foliar producto de la deficiencia de fósforo, como consecuencia de la reducción de la conductancia estomática. Del mismo modo, en plántulas de $P$. radiata la deficiencia de fósforo causó una reducción en el número de acículas y en el área foliar total (Conroy et al., 1986), fenómeno corroborado por Conroy et al. (1990), quienes determinaron que adecuadas concentraciones de fósforo (100 Kg/ha) incrementan el crecimiento de las plantas, principalmente por el aumento en la tasa de asimilación de $\mathrm{CO}_{2}$.

Estacas recolectadas desde plantas sometidas a los tratamientos $100 \mathrm{mg} / \mathrm{L}$ de N $/ 50 \mathrm{mg} / \mathrm{L}$ de P; 100 $\mathrm{mg} / \mathrm{L}$ de $\mathrm{N} / 0 \mathrm{mg} / \mathrm{L}$ de $\mathrm{P}$ obtuvieron altos porcentajes de enraizamiento $(76 \pm 2,0 \%)$. Esto se debe principalmente a los niveles de nitrógeno incorporados al medio de crecimiento, que permiten obtener estacas que forman callos en un menor período y que enraízan de forma apropiada. En raulí se encontró 
una relación entre los niveles de nitrógeno incorporados al medio de crecimiento y el enraizamiento adventicio, por ello la relación de carbohidrato y nitrógeno pueden ser manipuladas por las variaciones en las concentraciones de fertilizante. Se debe tener en cuenta que la nutrición nitrogenada afecta, los contenidos de hidrato de carbono, las concentraciones de nitrógeno en los tejidos (Moe \& Andersen, 1988), la producción de brotes y el enraizamiento adventicio. Una alta relación carbohidratos/nitrógeno promueve el enraizamiento y un cambio en esta relación puede afectar la respuesta rizogénica (Moe \& Andersen, 1988). La nutrición nitrogenada de setos de Pino taeda afecta los niveles de carbohidratos, la producción de vástagos y el enraizamiento adventicio. Los carbohidratos influyen en el enraizamiento debido a que proporcionan energía de reserva y estructuras de carbono para apoyar la iniciación y crecimiento de raíces. Además, las altas concentraciones de nitrógeno tienden a fomentar el consumo de hidratos de carbono disponibles para aumentar el crecimiento. Esto sugiere que una baja relación de nitrógeno en relación con hidratos de carbono disponibles, da lugar a una tendencia de almacenamiento de hidratos de carbono y los fotosintatos serían dirigidos a la formación de raíces adventicias.

\section{CONCLUSIONES}

Una concentración de nitrógeno de $500 \mathrm{mg} / \mathrm{L}$ proporcionada al medio de crecimiento causó toxicidad en las plantas, producto del incrementó de la conductividad eléctrica hasta los $1.994 \pm 186 \mu \mathrm{S} / \mathrm{cm}$. Las concentraciones de fósforo empleadas $(0,50,150,250 \mathrm{mg} / \mathrm{L})$ no afectaron a la conductividad eléctrica.

Al aumentar las concentraciones en el medio de crecimiento sobre los $100 \mathrm{mg} / \mathrm{L}$ de nitrógeno y $150 \mathrm{mg} / \mathrm{L}$ de fósforo disminuye la fotosíntesis neta, lo que se traduce en una disminución en las tasas de crecimiento en altura, diámetro, biomasa y área foliar.

El aumento en las concentraciones de nitrógeno en el medio de crecimiento provocó una disminución en el número total de brotes de primer orden producidas, no existiendo un efecto en el número total de estacas de segundo orden.

Los niveles de $100 \mathrm{mg} / \mathrm{L}$ de N / 0 mg/L de P; $100 \mathrm{mg} / \mathrm{L}$ de N / 50 mg/L mostraron los mejores resultados respecto al porcentaje de enraizamiento en estacas de primer orden. No se encontró un efecto de la fertilización en el enraizamiento de estacas de segundo orden.

\section{REFERENCIAS}

Bigg W.L. \& Schalau, J.W. (1990). Mineral nutrition and the target seedling. In: Rose, R., Campbell, S.J. \& Landis, T.D. (Eds). Proceedings of the Combined Meeting of the Western Forest Nursery Associations. Roseburg, Ore. 13-17 August, 1990. USDA Forest Service, Rocky Mountain Forest and Range Experiment Station. General Technical Report RM-200. Pp: 139-160.

Campbell, C.D. \& Sage, R.F. (2006). Interactions between the effects of atmospheric $\mathrm{CO}_{2}$ content and $\mathrm{P}$ nutrition on photosynthesis in white lupin (Lupinus albus L.). Plant Cell and Environment, 29. Pp: 844-853. https://doi.org/10.1111/j.1365-3040.2005.01464.x

Chang, S. (2003). Seedling sweetgum (Liquidambar styraciflua L.) halfsib family response to $N$ and $P$ fertilization: growth, leaf area, net photosynthesis and nutrient uptake. Forest Ecology and Management, 173. Pp: $281-291$. https://doi.org/10.1016/S0378-1127(02)00007-5

Comerford, N. \& Fisher, R. (1984). Using foliar analysis to classify nitrogen-deficient sites. Soil Science Society of America Journal, 48. Pp: 910-914. https://doi.org/10.2136/sssaj1984.03615995004800040042x

Conroy, J.P., Milham, P.J., Reed, M.L. \& Barlow, E.W. (1990). Increases in phosphorus requirements for $\mathrm{CO}_{2}-$ enriched pine species. Plant Physiology, 92. Pp: 977-982. https://doi.org/10.1104/pp.92.4.977

Conroy, J.P., Smillie, R.M., Küppers, M., Bevege, D.I. \& Barlow, E.W. (1986). Chlorophyll a fluorescence and photosynthetic and growth responses of Pinus radiata to phosphorus deficiency, drought stress, and high $\mathrm{CO}_{2}$. Plant Physiology, 81. Pp: 423-429. https://doi.org/10.1104/pp.81.2.423

Coombs, J., Hind, G., Leegood, R.C., Tieszen, L.L. \& Vonshak, A. (1985). Analytical Techniques. In: Coombs, J., Hall, D.O., Long, S.P. \& Scurlock, J.M.O. (Eds). Techniques in Bioproductivity and photosynthesis 2nd edition. Pergamon Press. Pp: 219-220. 
Domínguez, S., Oliet, J., Ruíz, L., Carrasco, I. \& Peñuelas, J. (2000). Influencia de la relación NPK en el desarrollo en vivero y campo de planta de Pinus pinea L. En: Actas del 1er Simposio del Pino Piñonero. Valladolid. 22-24 febrero, 2000.

Donoso, P., Monfil, T., Otero, L. \& Barrales, L. (1993). Estudio de crecimiento de plantaciones y renovales manejados de especies nativas en el área andina de las provincias de Cautín y Valdivia. Ciencia e Investigación Forestal 7: 253-287. https://doi.org/10.52904/0718-4646.1993.188

Donoso, P., Donoso, C., Marchelli, P., Gallo, L. \& Escobar, B. (2006). Nothofagus nervosa (Phil.) Dim. et Mil. En: Donoso, C. (Ed). Las especies arbóreas de los bosques templados de Chile y Argentina autoecología. Editorial Marisa Cuneo. Valdivia, Chile. Pp: 448-461.

Duryea, M.L. \& Mcclain, K. (1984). Altering seedling physiology to improve reforestation success. In: Duryea, M.L. \& Brown, G.N. (Eds). Seedling physiology and reforestation manual. Success I. Proceedings of the Physiology Working Group Technical Session. Oregon State University. Corvallis, Oregon. USA. Pp: 77-114. https://doi.org/10.1007/978-94-009-6137-1 5

Flugge, U., Freisl, M. \& Heldt, H. (1980). Balance between metabolite accumulation and transport in relation to photosynthesis by isolated spinach chloroplasts. Plant Physiology, 65. Pp: 574-577. https://doi.org/10.1104/pp.65.4.574

Hernández, A. \& Rubilar, P. (2012). Efecto de la fertilización nitrogenada y fosforada en el desarrollo y fenología de brotes de setos de Pinus radiata. Bosque, 33. Pp: 53-61. https://doi.org/10.4067/S0717-92002012000100006

Howell, K. \& Harrington, T. (2004). Nursery practices influence seedling morphology, field performance, and cost efficiency of containerized cherrybark oak. Southern Journal of Applied Forestry, 28. Pp: $152-162$. https://doi.org/10.1093/sjaf/28.3.152

Imo, M. \& Timmer, V. (1992). Nitrogen uptake of mesquite seedlings at conventional and experimential fertilization schedules. Soil Science Society of America Journal, 56. Pp: 927-934. https://doi.org/10.2136/sssaj1992.03615995005600030041x

Landis, T.D. (1985). Mineral nutrition as an index of seedling quality. En: Duryea, M. (Ed). Evaluating seedling quality: principles, procedures and predictive abilities of mayor test. Forest Research Lab. Oregon State University Corvaillis. Pp: 29-48.

Landis, T.D. (2000). Manual de viveros para la producción de especies forestales en contenedor. In Landis, T.D., Tinus, R.W., Mcdonald, S.E. \& Barnett, J.P. Manual Agricola. V. 4. Department of Agriculture, Forest Service. Washington DC. Pp: 1-67.

Lara, A., Cortés, M. \& Echeverría, C. (2000). Bosques. En: Sunkel, O. (Ed). Informe país: Estado actual del medio ambiente en Chile. Centro de Estudios de Política Pública. Universidad de Chile. Santiago. Pp: 131-173.

Loustau, D., Ben Brahim, M., Gaudillére, J.P. \& Dreyer, E. (1999). Photosynthetic responses to phosphorus nutrition in two-year-old maritime pine seedlings. Tree Physiology, $19 . \quad$ Pp: 707-715. https://doi.org/10.1093/treephys/19.11.707

Marschner, H. (1995). Mineral nutrition of higher plants, 2nd ed. Academic Press. London.

Moe, R. \& Andersen, A.S. (1988). Stockplant environment and subsequent adventitious rooting. En: Davies, T.D., Haissig, B.E. \& Sankula, N. (Eds). Adventitious Root Formation in Cuttings, Dioscorides, Portland, OR. Pp: 214-234.

Nicodemus, M., Salifu, F. \& Jacobs, D. (2008). Growth, nutrition, and photosynthetic response of Black walnut to varying nitrogen sources and rates. Journal of Plant Nutrition, 31. Pp: 1917-1936. https://doi.org/10.1080/01904160802402856

Oliet, J., Planelles, R., Artero, R.F., Valverde, R., Jacobs, D. \& Segura, M. (2009). Field performance of Pinus halepensis planted in Mediterranean arid conditions: relative influence of seedling morphology and mineral nutrition. New Forests, 37. Pp: 313-331. https://doi.org/10.1007/s11056-008-9126-3

Phillion, B.J. \& Bunting, W.R. (1983). Growth of spruce seedlings at various soluble fertilizer salt levels. Tree Plant Notes, 34. Pp: 31-33.

Radin, J. \& Eidenbock, M. (1984). Hydraulic conductance as a factor limiting leaf expansion of phosphorus-deficient cotton plants. Plant Physiology, 75. Pp: 372-377. https://doi.org/10.1104/pp.75.2.372

Reich, P., Oleksyn, J. \& Wright, I. (2009). Leaf phosphorus influences the photosynthesis-nitrogen relation: a crossbiome analysis of 314 species. Oecologia, 160. Pp: 207-212. https://doi.org/10.1007/s00442-009-1291-3 
Reyes, R., Gerding, V. \& Donoso, C. (2007). Crecimiento de una plantación de Nothofagus nervosa durante 20 años en Valdivia. Bosque, 28: Pp: 129-138. https://doi.org/10.4067/S0717-92002007000200005

Salisbury, F. \& Ross, C. (2000). Fisiología vegetal. Thomson Editores. Madrid. 988 p.

Sepúlveda, C. \& Stoll, A. (2003). Presencia de Nothofagus alpina (Poepp. et Endl.) Oerst. (Fagaceae) en el borde costero de la Región del Maule, Chile central. Gayana Botanica, 60. Pp: 134-135. https://doi.org/10.4067/S0717-66432003000200008

Salifu, K. \& Timmer, V. (2003). Optimizing nitrogen loading of Picea mariana seedlings during nursery culture. Canadian Journal Forest Research, 33. Pp: 1287-1294. https://doi.org/10.1139/x03-057

Silvak, M.N. \& Walker, D.A. (1986). Photosynthesis in vivo can be limited by phosphate supply. New Phytologist, 102. Pp: 499-512. https://doi.org/10.1111/j.1469-8137.1986.tb00826.x

Timmer, V. \& Armstrong, G. (1987). Growth and nutrition of containerized Pinus resinosa at exponentially increasing nutrient additions. Canadian Journal Forest Research, 17. Pp: 644-647. https://doi.org/10.1139/x87-105

Timmer, V. \& Parton, W. (1984). Optimum nutrient levels in a container growing medium determined by a saturation aqueous extract. Commun. Soil Science and Plant Analysis, $15 . \quad$ Pp: 607-618. https://doi.org/10.1080/00103628409367502

Thornton, F.C., Schaedle, M. \& Raynal, D.J. (1988). Sensitivity of red oak (Quercus rubra L.) and American beech (Fagus grandifolia Ehrh.) seedlings to sodium salts in solution culture. Tree Physiology, 4. Pp: 167-172. https://doi.org/10.1093/treephys/4.2.167

Van Den Driessche, R. (1991). Effects of nutrients on stock performance in the forest. En: Mineral nutrition of conifer seedlings. CRC Press. Florida, USA. Pp: 229-260. 